\title{
Asymptotic Freedom in Noninteger Dimensional Spaces
}

\author{
Subhash Kak \\ Oklahoma State University, Stillwater
}

\begin{abstract}
This paper shows that as the dimensionality of a noninteger dimensional falls below 2, the potential becomes constant irrespective of separation between objects and the force between them disappears, which represents a new paradigm of asymptotic freedom. Since asymptotic freedom is at the basis of many applications such as those of strange metals, unconventional superconductors, and fractional quantum Hall states, the new paradigm presented here can potentially have new and unexpected applications. It also is of relevance to the study of anomalous mechanical effects that are important in metamaterials.
\end{abstract}

\section{Introduction}

Asymptotic freedom [1,2,3] is a counterintuitive phenomenon [4] that plays a role in novel states of matter that include strange metals, unconventional superconductors $[5,6]$, and fractional quantum Hall states $[7,8,9]$. It describes the anomalous idea of strong interaction at large distances and much weaker interaction at short distances, which is associated with the behavior of quarks and gluons. As a property of some gauge theories, asymptotic freedom offers a mechanism for confinement at large distance by invoking properties of the mathematical structures used to describe these interactions.

Other counterintuitive phenomena are associated with negative differential response, and they are to be found not only in physics but also in chemistry and biological processes [10]. In materials that exhibit negative linear compressibility, there is expansion in one or more directions during the process of uniform compression $[11,12,13]$ and this anomalous mechanical behavior has found applications in the design of pressure sensors, artificial muscles and actuators $[14,15,16]$.

The counterintuitive nature of many quantum phenomena is understood through the lens of the complementarity principle [17], which provides a way to view seemingly inconsistent descriptions as two sides of the same reality. Although, the principle arose originally in quantum theory, it has been argued that it should apply much more broadly to different fields of science [18], including biological phenomena [19]. Complementarity is the description of the same phenomenon in distinct, categorically different ways that cannot be done in the same spatial, temporal, or situational context. 
Since the notion of dimension applies to physical reality at all conceivable scales, one must also consider anomalous mechanical properties of materials that emerges from compressing three-dimensional volumes into lower dimensions $[20,21,22]$, which may be seen as an example of increasing the energy scale, with prospective applications to superconductivity, ferroelectricity, information communication, sensing and detection $[23,24,25]$.

Some of the inconsistencies in the interpretation of phenomena could be the consequence of different ways space is considered in the theory. As stressed by Landauer [26], information is physical and, therefore, information-theoretic ideas must play a key role in the understanding of the above-mentioned anomalous phenomena.

I have recently shown $[27,28]$ that from the perspective of information theory, the optimum number of dimensions is the noninteger (and irrational) number $e$ and since Nature chooses optimality, this should be the dimensionality of physical space; it was also shown that the inverse square law itself may be seen as a consequence of noninteger dimensionality. The information efficiency per dimension is $E(d)=\frac{\ln d}{d}$ (Figure 1 shows the efficiency rise from zero for $d=1$, with peak at $e$ ) and the intrinsic dimensionality of data is also $e[29,30,31]$. Since dimensionality and information are basic concepts, these ideas ought to be of relevance in the analysis of counterintuitive phenomena listed above.

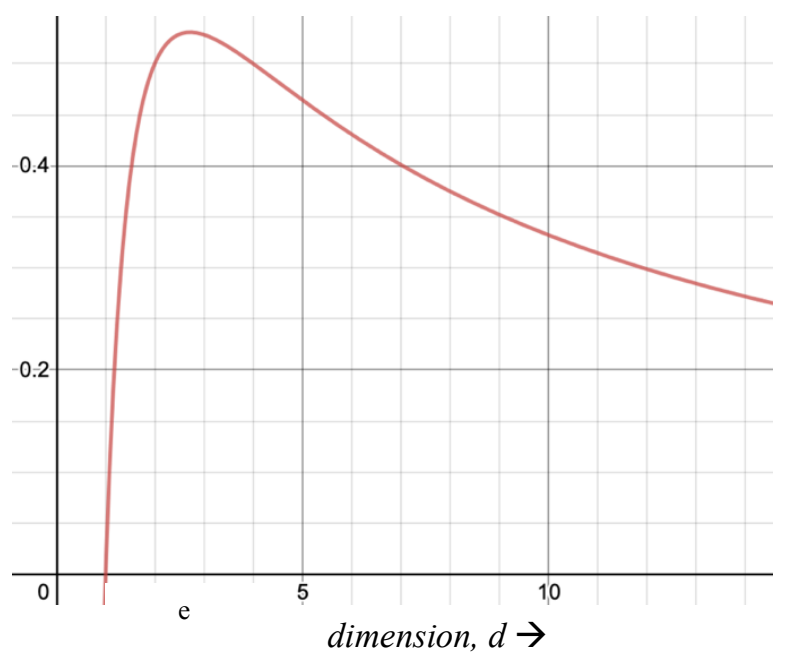

Figure 1. Dimensional efficiency is maximum at $d=e$

In this paper, rather than examining the application of noninteger dimensionality to any of the above phenomena directly, we show that a novel version of asymptotic freedom 
is associated with noninteger dimensionality. This represents a new framework with potential applications similar to those of anomalous mechanical properties of materials. Also, gauge transformations relate to the very nature of space, and so dimensionality may be of relevance to gauge theories.

\section{Dependence on $d$}

We can visualize a given noninteger space sitting within the container of the ceiling integer space (e.g. 1.7-dimensional space sits within the 2-dimensional space). It may be argued that a fundamental characteristic of a noninteger space is that of a continual shrinking of the metrical relationships between objects $[27,28]$. This is seen most clearly when we visualize a 2-dimensional space obtained from a 3-dimensional space which will cause all the points in the third dimension to collapse to the extant two dimensions.

Consider the example of a noninteger space with dimension $d$, which we shall compare with its ceiling function $\lceil d\rceil$, the smallest integer greater than it. Since dimensionality is an additive property, we can distribute the fractional part along any direction. Thus $d=$ 1.8 would imply a dimension of 0.9 along the one-dimensional infinite line in any direction.

The view of the noninteger dimension that there are gaps in the space is complementary to the view that relative measures on the line tend to shrink in proportion to the value of dimensionality, and the tendency to do so is a function of the ratio $\frac{(\lceil d\rceil-d)}{\lceil d\rceil}=\frac{1}{\lceil d\rceil} f(\lceil d\rceil-d)$, where $\lceil d\rceil$ is the ceiling function of $d$, and $f(\lceil d\rceil-d)$ represents the functional relationship with respect to the departure of the dimension value from its ceiling integer. For the example of $d=1.8$, the shrinking is a function of $\frac{1}{2} f(0.2)$.

The tendency for space to contract constitutes a potential, making points associated with events that the observer witnesses to tend to come closer to each other. This potential leads to dynamics that emerge thus from the very nature of space. This potential is not assumed to have an a priori existence, independent of dimensions, and therefore it represents a view different from the current understanding.

Since $d$ characterizes the space, the potential between two objects with unit measures must also be inversely related to the separation so that objects that are further apart have less influence on each other than those that are near. Points on a 1-dimensional infinite space stay where they are, whereas those on a 0.8-dimensional infinite space 
will have a potential to come closer by the 0.8 measure. Consider two objects with unit measures with a separation of $r$, and let the potential be defined by $p_{\lceil d\rceil}(d, r)$,

The sphere representing the equipotential surfaces at a distance of $r$ has surface equal to $S_{\lceil d\rceil}(d, r)$, but the distance $r$ should be modified to $\frac{d r}{\lceil d\rceil}$ because the density of the space along any line is smaller by the fraction $\frac{d}{|d|}$. Therefore, the potential will be proportional to

$$
p_{\lceil d\rceil}(d, r)=\frac{f(\lceil d]-d) d r}{\lceil d\rceil^{2}} \times \frac{1}{S_{[d]}(d, r)}=\frac{f(\lceil d]-d) d r}{[d\rceil^{2} S_{[d]}(d, r)}
$$

The value of $S_{d}$ for 3- and 2- and 1-dimensional worlds is $4 \pi r^{2}, 2 \pi r$, and 0 , respectively, and these are the surface area of a sphere, the circumference of a circle, and the length of a point. Corrected for reduced density, the expression for $S_{3}(d, r)$, and $S_{2}(d, r)$ becomes:

$$
S_{3}(d, r)=\frac{4}{9} \pi r^{2} d^{2} ; \quad S_{2}(d, r)=\frac{2 \pi r d}{2}=\pi r d
$$

The potentials for the ranges $2<d<3$ and $1<d<2$, that is $p_{3}(d, r)$ and $p_{2}(d, r)$, will be:

$$
p_{3}(d, r)=\frac{f(3-d)}{4 \pi r d} ; \quad p_{2}(d, r)=\frac{f(2-d)}{4 \pi}
$$

Note that $p_{2}(d, r)$ is independent of $r$.

The forces corresponding to these potentials will be the derivative of the expression (3). For a fixed $d$, over the range $2<d<3$, the potential is proportional to $1 / r$ and therefore the forces between objects in such a space will be proportional to $1 / r^{2}$. This is the origin of the inverse square law $[27,28]$.

The potentials as functions of dimensionality alone (in which case we simply drop $r$ as a variable) are:

$$
p_{3}(d)=\frac{f(3-d)}{4 \pi d} ; \quad p_{2}(d)=\frac{f(2-d)}{4 \pi}
$$

We need a theoretical framework to find these functions. In what follows, we use mathematical constraints to propose some candidates for these functions. 


\section{The function $p_{3}(d)$}

Some further constraints are needed to find plausible candidates for $f(3-d)$. This function should be zero for the values just outside the interval $2<d<3$ at $d=2$ and 3. The functions should be universal for unconstrained systems (as in cosmology), but would depend on external constraints for an engineered system.

A matched distribution. If physical structures are compared to the optimal filter corresponding to natural distribution, then the use of optimum signal theory [32] will require that the potentials be matched to the efficiencies associated with the $d$-values.

One can associate a pdf with the dimension $d$ and call it the DED (dimensional efficiency distribution) $f(d)$ with the range from 1 to the maximum of $N$ :

$$
f(d)=\frac{2}{(\ln N)^{2}} \frac{\ln d}{d}, \quad 1 \leq d \leq N
$$

where the factor $\frac{2}{(\ln N)^{2}}$ is to ensure that the area under it is 1 . Its expectation is $E(D)=$ $\frac{2}{(\ln N)^{2}}(N \ln N-N+1)$, and its variance is $\operatorname{VAR}(D)=\frac{2}{(\ln N)^{2}}\left(\frac{\ln N}{2} N^{2}-\frac{N^{2}}{4}+\frac{1}{4}\right)-$ $E(N)^{2}$.

The value of $N$ that is of interest to those looking at physical dimensions is 3. For such a case $\frac{2}{(\ln N)^{2}} \cong 1.658$ and $E(D) \cong 2.148$ and $\operatorname{VAR}(D) \cong 0.267$. Although the optimal value of dimensionality is $e$, the expected value of dimension is substantially less at 2.148 with a variance of 0.267 .

If $N=e, E(D)=2$ and $\operatorname{VAR}(D) \cong 0.195$. One can define a generalization of the DED as follows: $f_{\text {gen }}(a, d)=\frac{2 a}{(\ln a N)^{2}} \frac{\ln a d}{d}, 1 \leq d \leq N$, with the discrete version $p(n)=$ $\frac{1}{K} \frac{\ln a n}{n}, \quad 1 \leq n \leq N \quad$ where $K=\sum_{i=1}^{N} \ln (i a)^{1 / i}$.

To return to the idea of the $d$-dimensional space acting as a matched filter, one may choose the following function:

$$
f_{A}(3-d)=(d-2)(3-d) \frac{\ln d}{d}
$$

with $p_{3, A A}(d) \propto(d-2)(3-d) \frac{\ln d}{d^{2}}$, which is shown in Figure 2. 


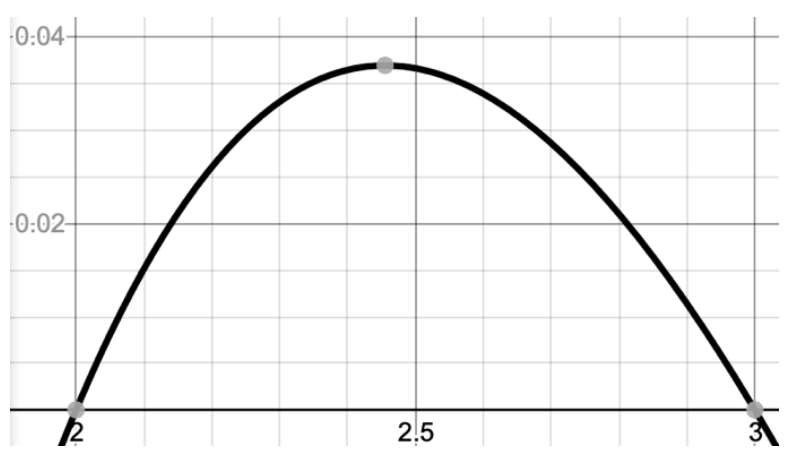

Figure 2. Potential $p_{3, C}(d)$ with peak at 2.455

Now we consider two ad hoc functions that satisfy the above constraints. The first of these is linear with respect to the constraints at 2 and 3 (which are multiplied); the second is linear with respect to 2 and exponential with respect to 3 with exponent of 0.25 .

$$
\begin{aligned}
& f_{B}(3-d)=(d-2)(3-d) \\
& f_{C}(3-d)=(d-2)\left(e^{(3-d)}-1\right)^{1 / 4}
\end{aligned}
$$

The potential function will then have the following characteristics:

$$
\begin{aligned}
& p_{3, B}(d)=\frac{(d-2)(3-d)}{4 \pi d} \\
& p_{3, C}(d)=\frac{(d-2)\left(e^{(3-d)}-1\right)^{1 / 4}}{4 \pi d}
\end{aligned}
$$

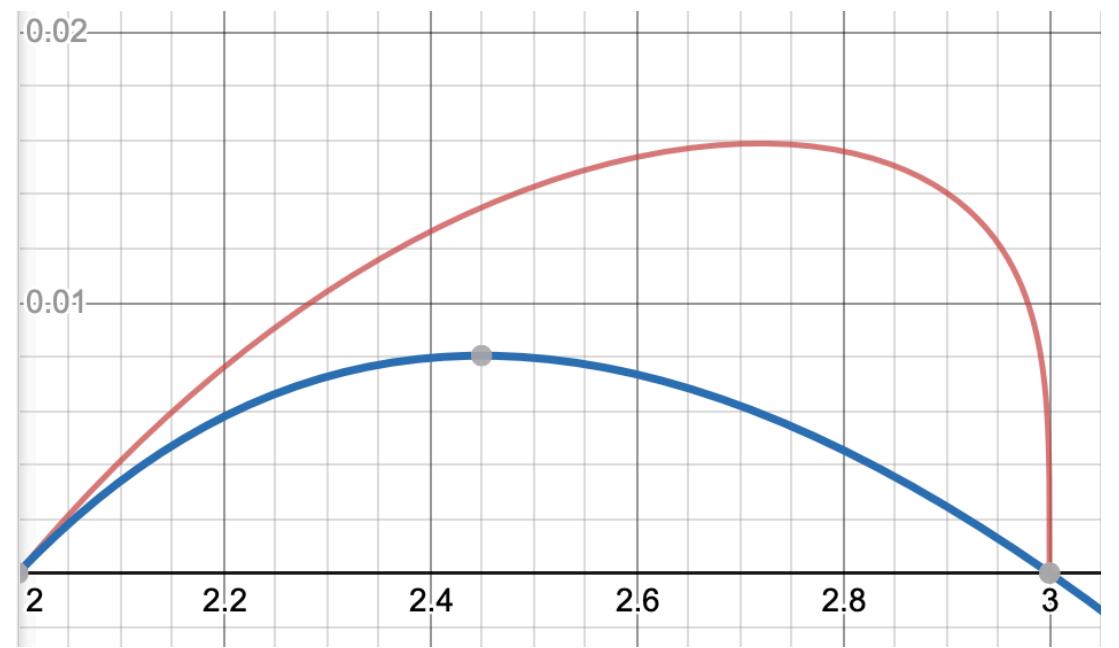

Figure 3. Potential with respect to dimensionality. Blue line $p_{3, B}(d)$, and Red line $p_{3, C}(d)$ 
The peak for the blue curve (equation 8 ) is at $d=2.4495$, whereas the peak for the red curve (equation 9 ) is at $d=2.7195$. This latter maximum is quite close to the optimal value of $e$.

\section{Asymptotic freedom for $1<d<2$}

The continuing reduction of dimensionality may be seen as injection of energy into the system as is done for engineered applications. The potential function $p_{2}(d, r)=\frac{f(2-d)}{4 \pi}$ being independent of $r$ implies that objects in such a space will experience the same potential and, therefore, will behave as if in a bag, and experience no force that varies with the separation. There will be a dependence on $d$, that can be put to use in engineering applications.

Since there is no dependence with respect to separation, this case represents emergence of freedom arrived at by squeezing the dimensionality of space.

Although there is no noninteger space generated intrinsic dynamics for the case of $1<d<2$, there could be dynamics as a consequence of externally applied forces.

\section{Conclusions}

This paper explored properties of noninteger dimensionality and examined how interaction potentials across objects vary. It was shown that as the dimensionality falls below 2 , the potential becomes constant, irrespective of separation, and the force between objects disappears, which represents a case of asymptotic freedom. We have shown that objects in a 2-dimensional space will not interact with each other, but as the dimensions increase beyond 2, the interactions will become strong. Systems could be engineered where the difference in these regimes is exploited to perform specific signal processing or anomalous mechanical function.

The work in this paper provides a dimensionality-based explanation of interactions becoming weaker as energy scales increase. Whether this phenomenon based on dimensionality of space has any connection with other models of asymptotic freedom remains to be investigated.

\section{REFERENCES}

1. Gross, D.J. Asymptotic freedom. Physics Today 40, 1, 39 (1987).

2. Gross, D.J. and Wilczek, F., Ultraviolet behavior of non-abelian gauge theories. Physical Review Letters 30, 1343-1346 (1973). 
3. Politzer, H.D. Reliable perturbative results for strong interactions. Physical Review Letters 30, 1346-1349 (1973).

4. Shore, B.W. Examples of counter-intuitive physics, Contemporary Physics 36, 15-28 (1995)

5. Keimer, B., Kivelson, S., Norman, M. et al. From quantum matter to high-temperature superconductivity in copper oxides. Nature 518, 179-186 (2015).

6. Zaanen, J. Why the temperature is high. Nature 430, 512-513 (2004).

7. Stoudenmire, E. M. \& White, S. R. Studying two-dimensional systems with the density matrix renormalization group. Annu. Rev. Condens. Matter Phys. 3, 6.1-6.18 (2012).

8. da Silva Neto, E. H. et al. Ubiquitous interplay between charge ordering and hightemperature superconductivity in cuprates. Science 343, 393-396 (2014)

9. Fujita, K. et al. Simultaneous transitions in cuprate momentum-space topology and electronic symmetry breaking. Science 344, 612-616 (2014)

10. Falasco, G. et al. Negative differential response of chemical reaction currents. New Journal of Physics 21 (2019).

11. Baughman, R. H., Stafstrom, S., Cui, C. X. \& Dantas, S. O. Materials with negative compressibilities in one or more dimensions. Science 279, 1522-1524 (1998).

12. Nicolaou, Z. G. \& Motter, A. E. Mechanical metamaterials with negative compressibility transitions. Nat. Mater. 11, 608-613 (2012).

13. Jiang, X., Molokeev, M.S., Dong, L. et al. Anomalous mechanical materials squeezing three-dimensional volume compressibility into one dimension. Nat Commun 11, 5593 (2020).

14. Frenzel, T., Kadic, M. \& Wegener, M. Three-dimensional mechanical metamaterials with a twist. Science 358, 1072-1074 (2017).

15. Fang, N. et al. Ultrasonic metamaterials with negative modulus. Nat. Mater. 5, 452-456 (2006).

16. Uhoya, W. et al. Anomalous compressibility effects and superconductivity of EuFe2As2 under high pressures. J. Phys.: Condens. Matter 22, 292202 (2010).

17. Bohr, N. Atomic theory and the description of nature. Cambridge University Press, Cambridge UK. (1934).

18. Bohr, N. Essays 1958-1962 on Atomic Physics and Human Knowledge. Wiley, New York. (1963)

19. Pattee, H.H. The complementarity principle in biological and social structures. Journal of Social and Biological Structures 1, 191-200 (1978).

20. Lee, Y., Vogt, T., Hriljac, J. A., Parise, J. B. \& Artioli, G. Pressure-induced volume expansion of zeolites in the natrolite family. J. Am. Chem. Soc. 124, 5466-5475 (2002).

21. Vakarin, E. V., Duda, Y. \& Badiali, J. P. Negative linear compressibility in confined dilatating systems. J. Chem. Phys. 124, 144515 (2006).

22. Grima, J. N., Attard, D. \& Gatt, R. Truss-type systems exhibiting negative compressibility. Phys. Status Solidi B 245, 2405-2414 (2008).

23. Lakes, R. S., Lee, T., Bersie, A. \& Wang, Y. C. Extreme damping in composite materials with negative-stiffness inclusions. Nature 410, 565-567 (2001).

24. Scheibner, C., Souslov, A., Banerjee, D. et al. Odd elasticity. Nat. Phys. 16, 475-480 (2020).

25. Balogh, S.G., Palla, G., Pollner, P. et al. Generalized entropies, density of states, and nonextensivity. Sci Rep 10, 15516 (2020). 
26. Landauer, R. The physical nature of information. Phys. Lett. A 217, 188-193 (1996).

27. Kak, S. Information theory and dimensionality of space. Scientific Reports (2020)

28. Kak, S. Noninteger Dimensional Spaces and the Inverse Square Law. (2020); TechRxiv: https://www.techrxiv.org/articles/preprint/Noninteger Dimensional Spaces and the In verse Square Law/13079720

29. Kak, S. The base-e representation of numbers and the power law. Circuits Syst. Signal Process. (2020) https://doi.org/10.1007/s00034-020-01480-0

30. Kak, S. Information, representation, and structure. International Conference on Recent Trends in Mathematics and Its Applications to Graphs, Networks and Petri Nets, New Delhi, India (2020).

31. Kak, S. The intrinsic dimensionality of data. Circuits Syst. Signal Process. (2020); https://doi.org/10.1007/s00034-020-01583-8

32. Turin G L. An introduction to matched filters. IRE Trans. Inform. Theory IT-6, 311- 329 (1960). 